\title{
Pterostilbene attenuates the proliferation and differentiation of TNF- $\alpha$-treated human periodontal ligament stem cells
}

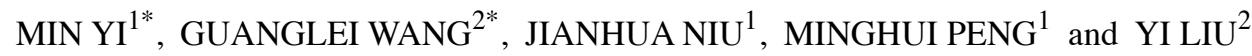 \\ ${ }^{1}$ Department of Integrative Therapy, Shanghai Huangpu District 2nd Dental Disease Prevention and Treatment Institute, \\ Shanghai 200001; ${ }^{2}$ Department of Stomatology, Jiading District Central Hospital Affiliated to \\ Shanghai University of Medicine and Health Sciences, Shanghai 201800, P.R. China
}

Received September 30, 2021; Accepted January 5, 2022

DOI: $10.3892 /$ etm.2022.11233

\begin{abstract}
Periodontitis is a common chronic inflammatory oral disease. The objective of periodontal treatment is to control infection whilst regenerating damaged periodontal tissue. The present study aimed to determine the potential effects of pterostilbene (PTE), a representative stilbene compound, on the proliferation and differentiation of human periodontal ligament stem cells (hPDLSCs). Different concentrations (1, 5, 10 and $20 \mu \mathrm{M}$ ) of PTE were applied to hPDLSCs, after which Cell Counting Kit- 8 and western blotting assays were performed to examine the protein levels of Ki67, PCNA, p-IкB $\alpha, \mathrm{I} \kappa \mathrm{B} \alpha$, $\mathrm{Bcl}-2$, Bax, cleaved caspase3. The effect of PTE on the release of inflammatory factors, including IL-1 $\beta$, IL-6 and IL-10 was assessed by RT-qPCR. The apoptosis of TNF- $\alpha$-induced hPDLSCs was evaluated by TUNEL assay and western blotting. Additionally, the role of PTE in hPDLSC mineralization was evaluated using alizarin red staining. The expression levels of mineralization indices, including RUNX2 and ALP were subsequently determined using western blotting. Subsequently, the target of PTE was predicted using TargetNet database and AutoDock v4.2 software and verified using western blotting. The results of the present study revealed that PTE promoted the proliferation of hPDLSCs in a concentration-dependent manner. Furthermore, PTE treatment decreased the release of inflammatory factors and alleviated the apoptosis of TNF- $\alpha$-induced hPDLSCs. PTE was also demonstrated to promote the formation of mineral nodules in TNF- $\alpha$-induced hPDLSCs. The Targetnet database, along with molecular docking, indicated that histone deacetylases (HDACs) were
\end{abstract}

Correspondence to: Dr Yi Liu, Department of Stomatology, Jiading District Central Hospital Affiliated to Shanghai University of Medicine and Health Sciences, 1 Chengbei Road, Shanghai 201800, P.R. China

E-mail: liuyily120@163.com

${ }^{*}$ Contributed equally

Key words: pterostilbene, proliferation, differentiation, human periodontal ligament stem cells, periodontitis the probable targets of PTE upstream of regulating periodontitis. The results of western blotting implied that TNF- $\alpha$ significantly increased expression levels of HDAC2, 4, 6 and 8, whilst PTE treatment markedly decreased HDAC4, 6 and 8 expression in a concentration-dependent manner compared with the TNF- $\alpha$ group, which further confirmed these conclusions. In summary, results of the present study revealed that PTE promoted TNF- $\alpha$-induced hPDLSC proliferation and differentiation, whilst alleviating inflammation and apoptosis. PTE also inhibited the expression of HDACs, which may be involved in the mechanism of periodontitis.

\section{Introduction}

Periodontitis is a common chronic inflammatory oral disease (1). With the gradual onset of inflammation, periodontitis causes destruction of the periodontal ligament, cementum and alveolar bone (2). If treatment is not applied in a timely manner, periodontitis will cause further periodontal detachment and bone loss, eventually leading to early tooth loss (3). Due to the importance of periodontal tissues, the objective of periodontal treatment is to first control the infection and inhibit disease progression, then to regenerate the damaged periodontal tissue and reconstruct the missing tooth support structures (4). At present, conventional treatments for periodontal disease are relatively basic in nature, including cleansing, curettage and root leveling (5). Although these can control inflammation and prevent the progression of periodontal disease, they cannot completely restore the damaged tooth support structure (5). In addition, although the emergence of guided tissue regeneration methods has improved the treatment of periodontal disease, its ultimate effect remains limited and unstable $(6,7)$. Furthermore, indications for guided tissue regeneration must be strictly screened and clinical predictability of its application is poor, limiting its application further $(6,7)$. Over the previous decade, with the development of regenerative medicine, stem cell-based tissue engineering technology has provided an important means of repairing damaged tissues whilst preserving the highly ordered surrounding internal environment (8-11). This observation has been reported by a number of previous preclinical studies (8-11). In particular, human periodontal ligament stem cells (hPDLSCs) have been demonstrated to favorably regenerate supporting tissues 
surrounding the tooth in vivo (12). Therefore, hPDLSCs are increasingly being applied for the regeneration treatment of bone loss caused by periodontal disease (12).

Stilbene compounds are general names given to a series of compounds with the stilbene group as the parent backbone, which serve important roles in pharmacology. For example, several stilbene-based drugs such as raloxifene, toremifene and tamoxifen, have been approved clinically for treating various diseases, including breast cancer (13). Pterostilbene (PTE; 3, 5-dimethoxy-4'-hydroxystilbene) is a representative of stilbene compounds that can be found naturally in blueberries and red sandalwood (14). PTE is a methylated derivative of resveratrol, both of which confer considerable anti-oxidant, anti-inflammatory and anticancer effects (15). On account of the presence of two methoxy groups, PTE has been reported to exert improved lipophilicity, increased oral absorption and higher bioavailability compared with resveratrol $(16,17)$. Therefore, it has been considered to be a potential drug for the treatment of a number of diseases, such as Alzheimer's disease, vascular dementia, bladder cancer $(16,17)$. Previous studies have revealed that PTE can prevent hypoxia-reoxygenation injury by activating sirtuin 1 in cardiomyocytes (18), in addition to improving cardiac function and reducing oxidative stress in animal models of ischemia-reperfusion injury (19). Another previous study suggested that PTE reduced high-fat-induced atherosclerosis in mice by inhibiting various proinflammatory cytokines, including TGF- $\beta$, TNF- $\alpha$ and interleukins (20). In particular, it was also demonstrated that PTE can reduce the release of inflammatory factors, including IL-1 $\beta$, IL- 6 and TNF- $\alpha$ by macrophages induced by oral symbiotic bacteria (21). Therefore, the present study hypothesized that PTE can prevent the onset of periodontitis.

TNF- $\alpha$ is one of the pivotal endogenous mediators of periodontitis (22). Therefore, TNF- $\alpha$ was used to induce hPDLSCs in the present study to assess the effects of PTE on cell proliferation and differentiation. Results of the present study may expand the current understanding of the efficacy of PTE and provide a novel direction for the design of treatment strategies for periodontitis.

\section{Materials and methods}

Cell culture and grouping. hPDLSCs (cat. no. BJ-ATCC0562; primary cells; Shanghai Bangjing Industry Co., Ltd.) were cultured in $\alpha$-MEM (Gibco; Thermo Fisher Scientific, Inc.) supplemented with 10\% FBS (Invitrogen; Thermo Fisher Scientific, Inc.) and $1 \%$ penicillin/streptomycin (Invitrogen; Thermo Fisher Scientific, Inc.) in $5 \% \mathrm{CO}_{2}$ at $37^{\circ} \mathrm{C}$.

PTE (purity $\geq 97 \%$; Sigma-Aldrich; Merck KGaA; Fig. 1A) was dissolved in DMSO (purity $>99.8 \%$; Shanghai Aladdin Biochemical Technology Co., Ltd.) and diluted to concentrations of $1,5,10$ and $20 \mu \mathrm{M}$ (23). TNF- $\alpha$ (purity $\geq 95 \%$; Sigma-Aldrich; Merck KGaA) was dissolved in DMSO and diluted to concentration of $100 \mathrm{ng} / \mathrm{ml}$ (24). Following the determination of PTE concentration, hPDLSCs were divided into the following five groups: i) Control (untreated); ii) TNF- $\alpha$ (100 ng/ml), iii) PTE low (L; TNF- $\alpha+$ PTE low dose $5 \mu \mathrm{M})$; iv) PTE medium (M; TNF- $\alpha+$ PTE medium dose $10 \mu \mathrm{M})$; and v) PTE high (H; TNF- $\alpha+$ PTE high dose $20 \mu \mathrm{M})$.
Bioinformatics analysis. TargetNet (www.targetnet.scbdd.com) is a platform that can be used for drug target prediction (25). The structure of PTE was input with default parameters (area under curve $\geq 0.7$; fingerprint type: extended connectivity fingerprint 4) to obtain a report of probable target proteins.

Molecular docking technology is a theoretical simulation method used for studying the interactions among molecules, such as ligands and receptors, in addition to predicting the nature of their binding mode and affinity (26). The structure of PTE was first imported into the OpenBabel v2.2.1 software (Free software foundation, Inc.) for hydrogenation and conversion into its 3D structure. The 3D structure of various histone deacetylase (HDAC) proteins, including HDAC2 (PDB ID: 6WBW), HDAC4 (PDB ID: 6FYZ), HDAC6 (PDB ID: 5EDU) and HDAC8 (PDB ID: 5D1B), were downloaded from the Protein Data Bank website (https://www.rcsb.org/). To reduce the influence of binding force prediction, water molecules and existing ligands in the protein structure were deleted using PyMOL v2.2.0 software (DeLano Scientific, LLC). The position of the original ligand was set as a docking site before the docking between PTE and HDACs was performed using AutoDock v4.2 software (Scripps Institute) via automatic calculation of binding free energy PTE and HDACs in the running process of the system. All docking calculations were performed using a Lamarckian genetic algorithm. The default FlexX parameters were used.

Cell Counting Kit-8(CCK-8) assay. Cell viability was measured using CCK-8 assay kit (Dojindo Molecular Technologies, Inc.). hPDLSCs were seeded into 96-well plates at a density of $3 \times 10^{3}$ cells/well. After allowing for cell adherence, the original culture medium was replaced with medium containing PTE $(0,1,5,10$ and $20 \mu \mathrm{M})$ and/or TNF- $\alpha(100 \mathrm{ng} / \mathrm{ml})$. After $48 \mathrm{~h}$ of incubation at $37^{\circ} \mathrm{C}, 10 \mu \mathrm{l} \mathrm{CCK}-8$ was added to each well, after which samples were incubated at $37^{\circ} \mathrm{C}$ for an additional $2 \mathrm{~h}$. Optical density (OD) values were recorded at $450 \mathrm{~nm}$ using a microplate reader (Dojindo Molecular Technologies, Inc.).

Alkaline phosphatase (ALP) activity assay. hPDLSCs were seeded into six-well plates at a density of $2 \times 10^{5}$ cells/well. After cells reached $60-70 \%$ confluence, the culture medium was replaced with osteogenesis induction medium [ $\alpha$-MEM supplemented with $10 \%$ FBS, $10 \mathrm{mM} \beta$-glycerolphosphate (China Pharmaceutical Shanghai Chemical Reagent Co., Ltd.), $50 \mathrm{mg} / 1$ ascorbate and $2 \mathrm{mg} / 1$ dexamethasone (both Sigma-Aldrich; Merck KGaA)] containing TNF- $\alpha(100 \mathrm{ng} / \mathrm{ml})$ and PTE $(0,5,10$ and $20 \mu \mathrm{M})$. Briefly, the cells were lysed with $0.05 \%$ Triton X-100 (Sigma-Aldrich) at $4^{\circ} \mathrm{C}$ for $2 \mathrm{~h}$. On day 7 following osteogenic induction at $37^{\circ} \mathrm{C}$, the cell lysate was centrifuged at $10,000 \mathrm{x}$ g for $15 \mathrm{~min}$ at $4^{\circ} \mathrm{C}$ to collect the supernatant. ALP activity was measured according to the manufacturer's protocol of the ALP activity detection kit (cat. no. P0321; Beyotime Institute of Biotechnology). The OD value was recorded at $405 \mathrm{~nm}$ using a microplate reader (Dojindo Molecular Technologies, Inc.).

Terminal deoxynucleotidyl-transferase-mediated dUTP nick end labeling (TUNEL) assay. The apoptosis of hPDLSCs cells was assessed using a TUNEL assay (Beyotime Institute of Biotechnology) according to the manufacturer's instructions. 
A<smiles>COc1cc(/C=C/c2ccc(O)cc2)cc(OC)c1</smiles>

Pterostilbene (PTE)

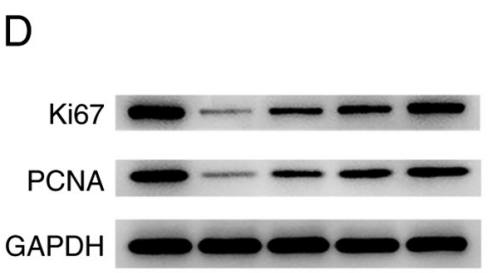

$\mathrm{B}$
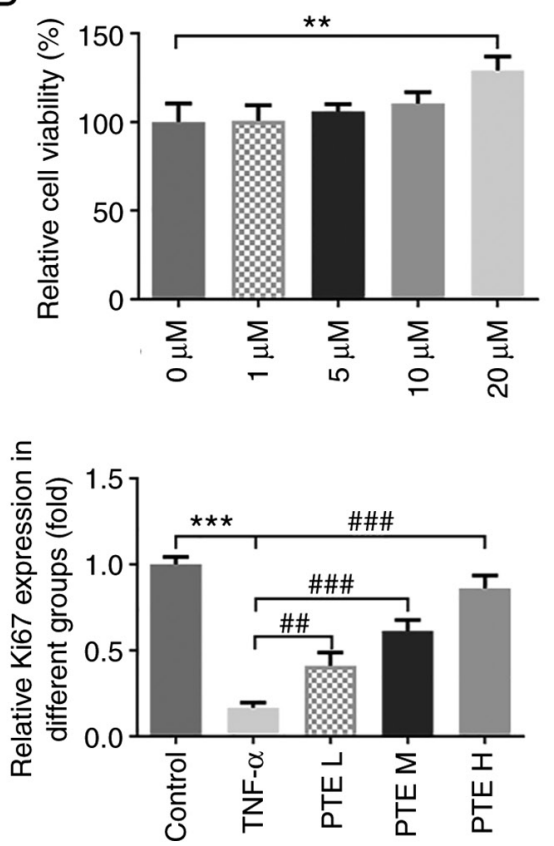

C
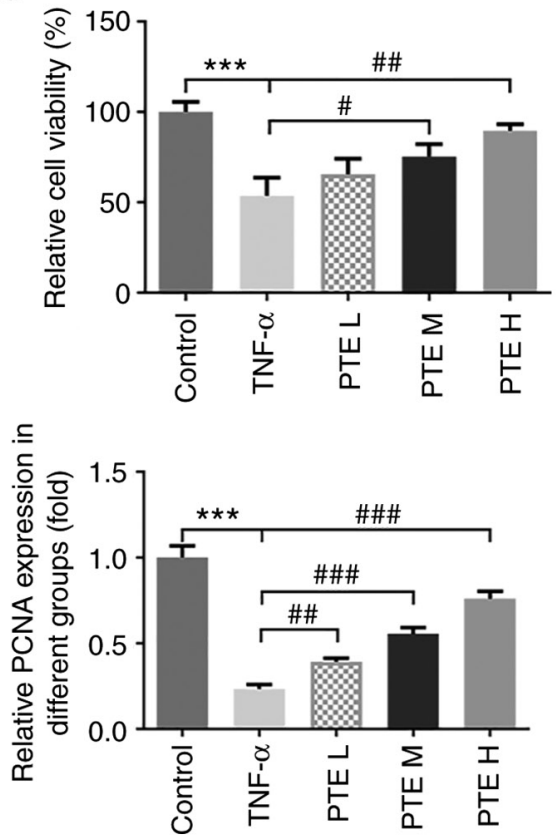

Figure 1. PTE promotes TNF- $\alpha$-induced hPDLSC proliferation. (A) Chemical structure of PTE. (B) Effect of varying concentrations of PTE on hPDLSC viability was determined using a CCK-8 assay. (C) Effect of different concentrations of PTE and TNF- $\alpha$ on hPDLSC viability was also assessed using a CCK-8 assay. (D) The expression levels of Ki67 and PCNA were measured using western blotting. ${ }^{* *} \mathrm{P}<0.01$ and ${ }^{* * *} \mathrm{P}<0.001 ;{ }^{\#} \mathrm{P}<0.05,{ }^{\# \prime} \mathrm{P}<0.01$ and ${ }^{\# \# \#} \mathrm{P}<0.001$. PTE, pterostilbene; hPDLSCs, human periodontal ligament stem cells; CCK-8, Cell Counting Kit-8; PCNA, proliferating cell nuclear antigen; L, low dose; $\mathrm{M}$, medium dose; $\mathrm{H}$, high dose.

After 24 h cell culture, transfected cells were fixed with $1 \%$ paraformaldehyde at room temperature for $15 \mathrm{~min}$ and $50 \mu \mathrm{l}$ TUNEL was used to incubate cells for $1 \mathrm{~h}$ at $37^{\circ} \mathrm{C}$, followed by staining of nuclear DNA with $10 \mu \mathrm{g} / \mathrm{ml}$ DAPI at $37^{\circ} \mathrm{C}$ for 2-3 min and mounted with glycerol gelatin (Sigma-Aldrich; Merck KGaA). The cells were analyzed from three fields of view using a fluorescence microscope (magnification, x200; Olympus Corporation).

Reverse transcription-quantitative PCR (RT-qPCR). hPDLSCs were incubated in six-well plates at a density of $2 \times 10^{5}$ cells/well. TNF- $\alpha(100 \mathrm{ng} / \mathrm{ml})$ and PTE $(0,5,10$ and $20 \mu \mathrm{M})$ were added to the plates at $37^{\circ} \mathrm{C}$ until cells reached $60-70 \%$ confluence. Cells were subsequently incubated at $37^{\circ} \mathrm{C}$ for $48 \mathrm{~h}$. For the determination of ALP and runt-related transcription factor 2 (RUNX2) expression levels, cells were collected on day 7 of osteogenic induction. Total RNA was extracted from the cultured cells using TRIzol ${ }^{\circledR}$ reagent (Invitrogen; Thermo Fisher Scientific, Inc.) according to the manufacturer's protocol and reversed transcribed into cDNA using the PrimeScript RT reagent kit (Takara Bio, Inc.) in accordance with the manufacturer's protocol. qPCR reactions were performed using the SYBR Green Master Mix (Biosharp Life Sciences) on a 7500 Real-time system (Applied Biosystems; Thermo Fisher Scientific, Inc.) according to the manufacturer's protocol. The thermocycling conditions were as follows: Initial denaturation at $95^{\circ} \mathrm{C}$ for $5 \mathrm{~min}$, followed by 45 cycles of $95^{\circ} \mathrm{C}$ for $30 \mathrm{sec}, 55^{\circ} \mathrm{C}$ for $30 \mathrm{sec}, 72^{\circ} \mathrm{C}$ for $30 \mathrm{sec}$ and $72^{\circ} \mathrm{C}$ for $7 \mathrm{~min}$. Relative mRNA expression was quantified using the $2^{-\Delta \Delta \mathrm{Cq}}$ method (27) and normalized to GADPH. The primer sequences used for qPCR are listed in Table I.
Western blotting. The treatment of hPDLSCs was the same as that described in RT-qPCR. Protein lysates from hPDLSCs were prepared using RIPA lysis buffer (Beyotime Institute of Biotechnology) and protein concentration was measured using a BCA kit (Beijing Solarbio Science \& Technology, Co., Ltd.). Samples (20 $\mu \mathrm{g}$ per lane) were subjected to 10 or $12 \%$ SDS-PAGE and transferred onto PVDF membranes. After blocking with 5\% non-fat milk at room temperature for $1 \mathrm{~h}$, blots were incubated with the following primary antibodies overnight at $4^{\circ} \mathrm{C}$ : $\mathrm{Ki} 67$ (1:100; cat. no. ab16667; Abcam), phosphorylated (p)-IкB $\alpha$ (1:1,000; cat. no. 2859; Cell Signaling Technology, Inc.), I $\kappa \alpha$ (1:1,000; cat. no. 4812; Cell Signaling Technology, Inc.), cleaved caspase 3 (1:500; cat. no. ab32042; Abcam), ALP (1:500, cat. no. ab65834; Abcam), runt-related transcription factor 2 (RUNX2; 1:1,000; cat. no. ab23981; Abcam), GAPDH (1:10,000; cat. no. ab181602; Abcam), proliferating cell nuclear antigen (PCNA; 1:1,000; cat. no. 13110; Cell Signaling Technology, Inc.), Bcl2 (1:1,000; cat. no. 4223; Cell Signaling Technology, Inc.), Bax (1:1,000; cat. no. 5023; Cell Signaling Technology, Inc.), HDAC2 (1:1,000; cat. no. 57156; Cell Signaling Technology, Inc.), HDAC4 (1:1,000; cat. no. 15164; Cell Signaling Technology, Inc.), HDAC6 (1:1,000; cat. no. 7558; Cell Signaling Technology, Inc.) and HDAC8 (1:1,000; cat. no. 66042; Cell Signaling Technology, Inc.). The membranes were then incubated with HRP-conjugated goat anti-rabbit (1:10,000; cat. no. ab6721; Abcam) secondary antibodies for $2 \mathrm{~h}$ at room temperature and developed using an ECL kit (Biosharp Life Sciences). ImageJ v1.8.0 software (National Institutes of Health) was applied for image analysis and relative protein expression was normalized to GAPDH. 
Table I. Sequences of the primers.

\begin{tabular}{llcc}
\hline Gene & \multicolumn{1}{c}{ Sequence (5'-3') } & $\begin{array}{c}\text { NCBI Reference } \\
\text { sequence }\end{array}$ & $\begin{array}{c}\text { Product } \\
\text { size }\end{array}$ \\
\hline $\begin{array}{l}\text { IL-1 } \beta \\
\text { Forward } \\
\text { Reverse }\end{array}$ & CTACCTGTCCTGCGTGTTGA & NM_000576.3 \\
IL-6 & GGGAACTGGGCAGACTCAAA & & 153 \\
Forward & CCTTCGGTCCAGTTGCCTTCT & NM_000600.5 & 233 \\
Reverse & CAGTGCCTCTTTGCTGCTTTC & & 148 \\
IL-10 & NM_000572.3 & \\
$\begin{array}{l}\text { Forward } \\
\text { Reverse }\end{array}$ & AGACAGACTTGCAAAAGAAGGC & & 231 \\
ALP & TCGAAGCATGTTAGGCAGGTT \\
Forward & CTTGTGCCTGGACGGACC & NM_000478.6 & 129 \\
Reverse & CGCCAGTACTTGGGGTCTTT & & 138 \\
Forward & CGAATGGCAGCACGCTATTAA & NM_001015051.4 & \\
Reverse & GTCGCCAAACAGATTCATCCA & & \\
GAPDH & GCACCGTCAAGGCTGAGAAC & NM_001256799.3 & \\
Forward & TGGTGAAGACGCCAGTGGA & & \\
\hline
\end{tabular}

ALP, alkaline phosphatase; RUNX2, runt-related transcription factor 2.

Alizarin red staining. hPDLSCs were seeded into 12-well plates at a density of $1 \times 10^{5}$ cells/well. After the cells reached $60-70 \%$ confluence, the culture medium was replaced with osteogenesis induction medium containing TNF- $\alpha(100 \mathrm{ng} / \mathrm{ml})$ and PTE $(0,5,10$ and $20 \mu \mathrm{M})$. At 21 days after osteogenic induction at $37^{\circ} \mathrm{C}$, cells were washed once with PBS and fixed with $4 \%$ paraformaldehyde for $20 \mathrm{~min}$ at room temperature. $0.04 \mathrm{M}$ Alizarin Red S staining solution (cat. no. C0148S; Beyotime Institute of Biotechnology) was subsequently added for $30 \mathrm{~min}$ at room temperature. Images were observed under a light microscope (magnification, x200; Olympus, Corporation).

Statistical analysis. All experimental data are presented as the mean \pm SD from three independent experiments. GraphPad Prism 8.0 statistical software (GraphPad Software, Inc.) was used to analyze the data. Additionally, the data were in accordance with the normal distribution by Shapiro-Wilk test. One-way ANOVA and Tukey's post hoc test was conducted to compare differences between groups. $\mathrm{P}<0.05$ was considered to indicate a statistically significant difference.

\section{Results}

PTE promotes TNF- $\alpha$-induced $h P D L S C$ viability and alleviates inflammation. The effect of different concentrations of PTE $(0,1,5,10$ and $20 \mu \mathrm{M})$ on the viability of hPDLSCs was determined using a CCK-8 assay. The results revealed that PTE at $20 \mu \mathrm{M}$ increased cell viability compared with control untreated cells (Fig. 1B). Since PTE at a concentration of $1 \mu \mathrm{M}$ did not have a significant effect on cells, PTE at 5, 10 and
$20 \mu \mathrm{M}$ concentrations were used in subsequent experiments and labeled as L, M and $\mathrm{H}$ groups, respectively. Following TNF- $\alpha$ induction, the effects of these three concentrations of PTE on cell viability were assessed using a CCK- 8 assay. The results indicated that cell viability was significantly decreased in the TNF- $\alpha$ group compared with the control group, but subsequent PTE treatment reversed this reduction in cell viability in a concentration-dependent manner (Fig. 1C). The expression levels of Ki67 and PCNA were then measured using western blotting. The data revealed that the expression levels of each protein were significantly decreased following TNF- $\alpha$ treatment. However, PTE significantly reversed these reductions in Ki67 and PCNA expression (Fig. 1D). These results suggested that PTE prevented hPDLSCs from TNF- $\alpha$-induced damage and upregulated cell viability.

The expression levels of certain inflammatory factors, including IL-1 $\beta$, IL-6 and IL-10, were next assessed in hPDLSCs by performing RT-qPCR. The results revealed that the levels of IL-1 $\beta$ and IL- 6 were significantly increased, whilst that of IL-10 was significantly decreased, in the TNF- $\alpha$ group compared with the control group. Furthermore, PTE treatment significantly downregulated IL-1 $\beta$ and IL- 6 levels whilst upregulating IL-10 expression compared with the TNF- $\alpha$ group (Fig. 2A). In addition, western blotting revealed that PTE inhibited the TNF- $\alpha$-induced phosphorylation of p65, with $\mathrm{M}$ and $\mathrm{H}$ groups reaching significance (Fig. 2B).

PTE reduces apoptosis and promotes TNF- $\alpha$-induced hPDLSC differentiation. Cell apoptosis was assessed using TUNEL and western blotting assays. The number of apoptotic cells 

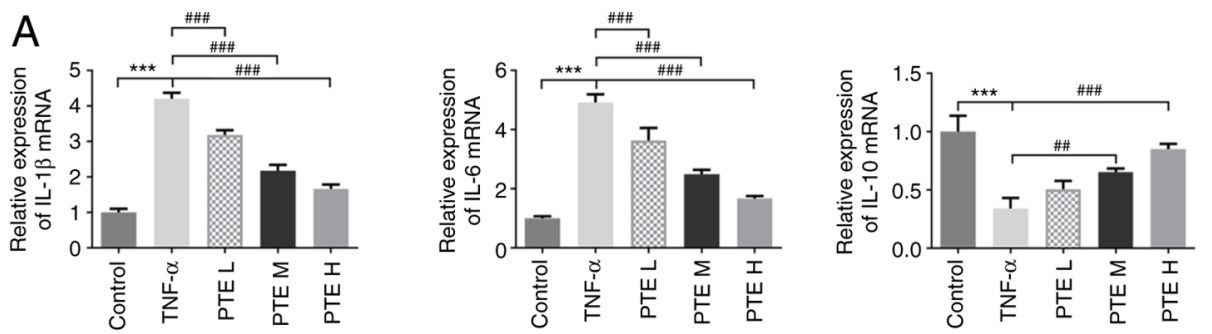

C
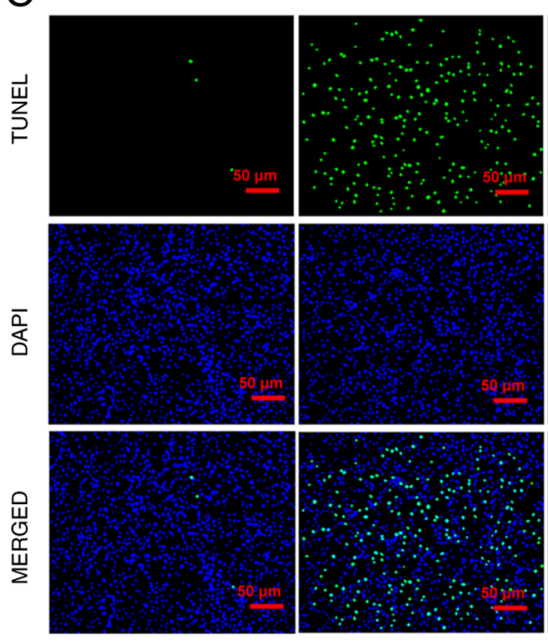

Control

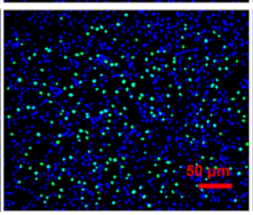

TNF- $\alpha$

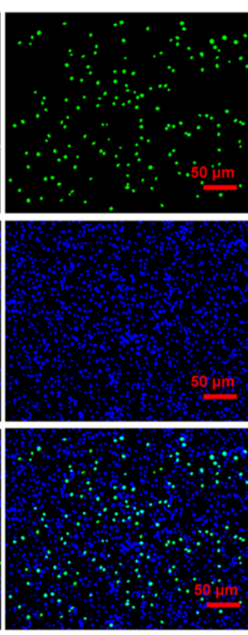

PTE L

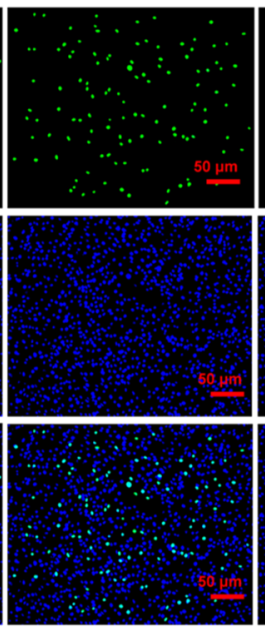

PTE M

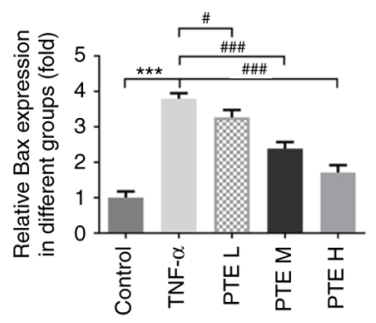

B
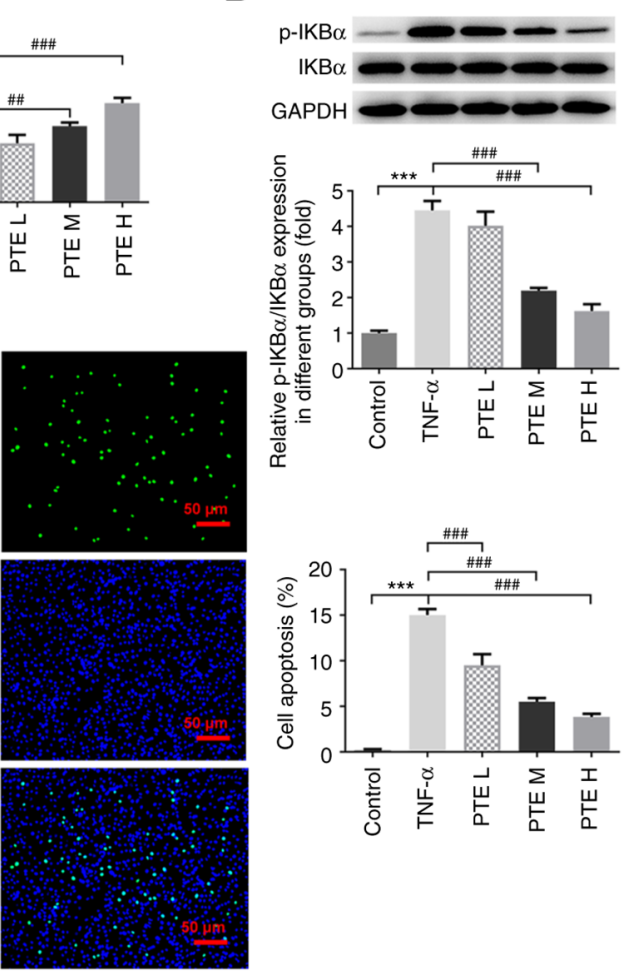

PTE H

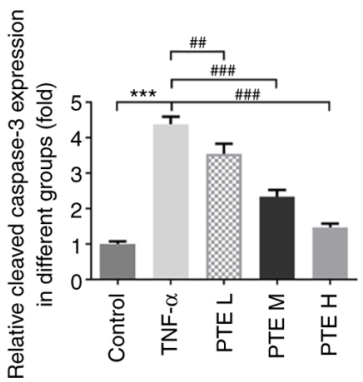

Figure 2. PTE alleviates TNF- $\alpha$-induced hPDLSC inflammation and apoptosis. (A) Levels of inflammatory factors IL-1 $\beta$, IL-6 and IL-10 were assessed in each group of hPDLSCs using reverse transcription-quantitative PCR. (B) Levels of I $\mathrm{B} \alpha$ and I $\mathrm{B} \alpha$ phosphorylation were determined using western blotting. (C) Cell apoptosis was assessed using a TUNEL assay. (D) The expression levels of Bcl2, Bax and cleaved caspase 3 were determined using western blotting. ${ }^{* * *} \mathrm{P}<0.001 ;{ }^{\#} \mathrm{P}<0.05,{ }^{\# \#} \mathrm{P}<0.01$ and ${ }^{\# \#} \mathrm{P}<0.001$. PTE, pterostilbene; hPDLSCs, human periodontal ligament stem cells; p-, phosphorylated; L, low dose; $\mathrm{M}$, medium dose; $\mathrm{H}$, high dose.

emitting green fluorescence in the TNF- $\alpha$ group was increased significantly compared with the control group. Additionally, as the PTE concentration increased, the number of apoptotic cells decreased significantly (Fig. 2C). Furthermore, the expression levels of Bax and cleaved caspase 3 were significantly increased in the TNF- $\alpha$ group compared with the control. However, they were significantly decreased in the PTE-treated groups compared with the TNF- $\alpha$ group (Fig. 2D). Bcl2 expression levels demonstrated the opposite trend following TNF- $\alpha$ and PTE treatment (Fig. 2D). The results of these assays suggested that PTE alleviated TNF- $\alpha$-induced hPDLSC apoptosis.

The role of PTE in the mineralization capacity of hPDLSCs was subsequently evaluated using alizarin red staining. While clear red mineralized nodules were observed in the control group, no staining could be observed in the TNF- $\alpha$ group. However, PTE treatment promoted the formation of mineral nodules in TNF- $\alpha$-induced hPDLSCs (Fig. 3A). The expression levels of mineralization indices were determined using western blotting and RT-qPCR. The protein and mRNA expression levels of ALP and RUNX2 were both significantly downregulated in the TNF- $\alpha$ group, which was significantly reversed in the three PTE groups (Fig. 3B and C). The activity of ALP was similarly decreased significantly by TNF- $\alpha$, which was also significantly reversed by all three doses of PTE (Fig. 3D).

HDACs are probable targets of PTE. The targets of PTE were predicted using the Targetnet database. The results determined HDAC2, 4, 6 and 8 to be probable targets of PTE. Therefore, the possible association between PTE and these proteins were calculated using the molecular docking module. The whole protein was presented in green, whilst the main skeleton of PTE was presented in brown. The blue solid line represented hydrogen bonds, the green dashed line represented $\pi$-stacking and the gray dashed line represented hydrophobic interactions. The docking values between PTE and HDAC2, 4, 6 and 
A

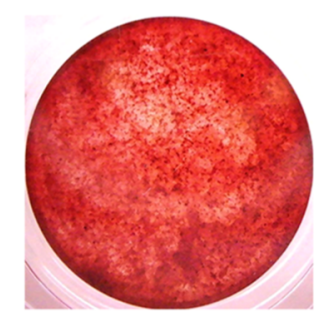

Control

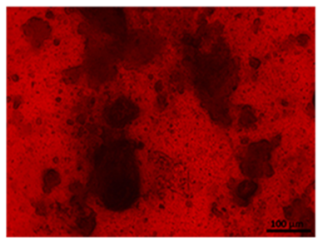

Control

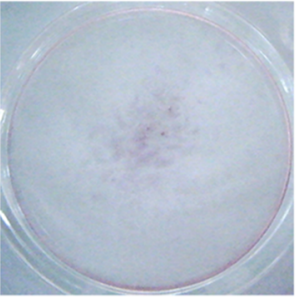

TNF- $\alpha$

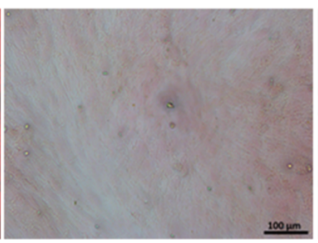

TNF- $\alpha$

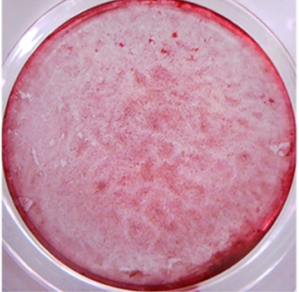

PTE L

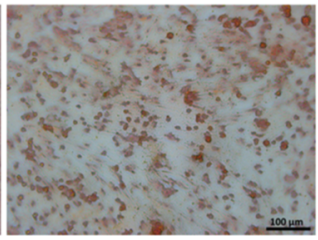

PTE L

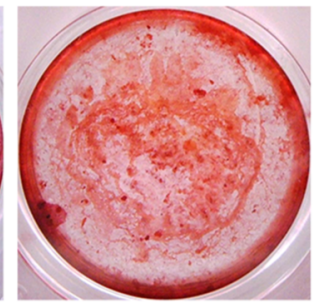

PTE M

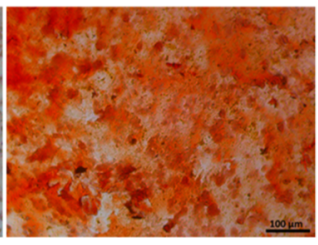

PTE M

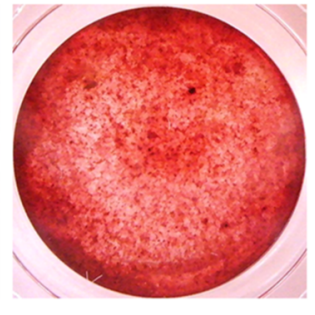

PTE H

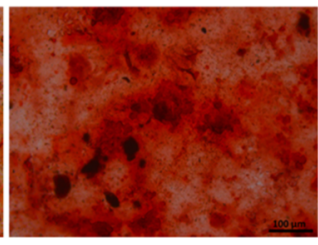

PTE H
B
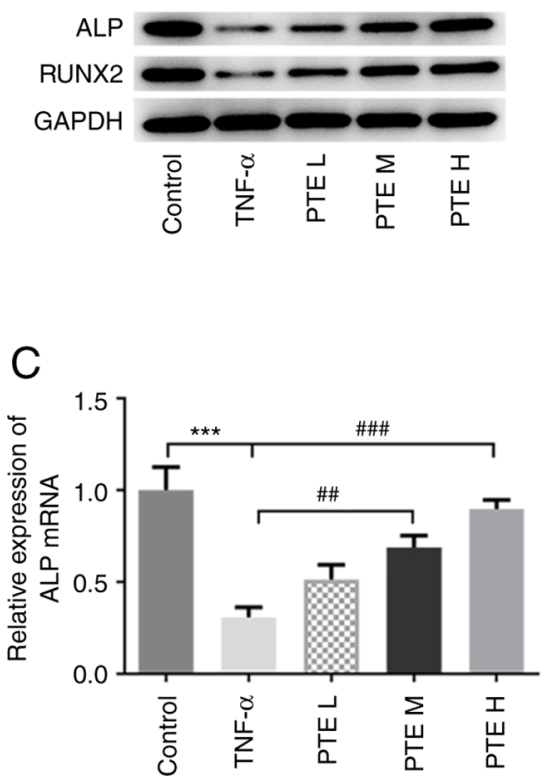
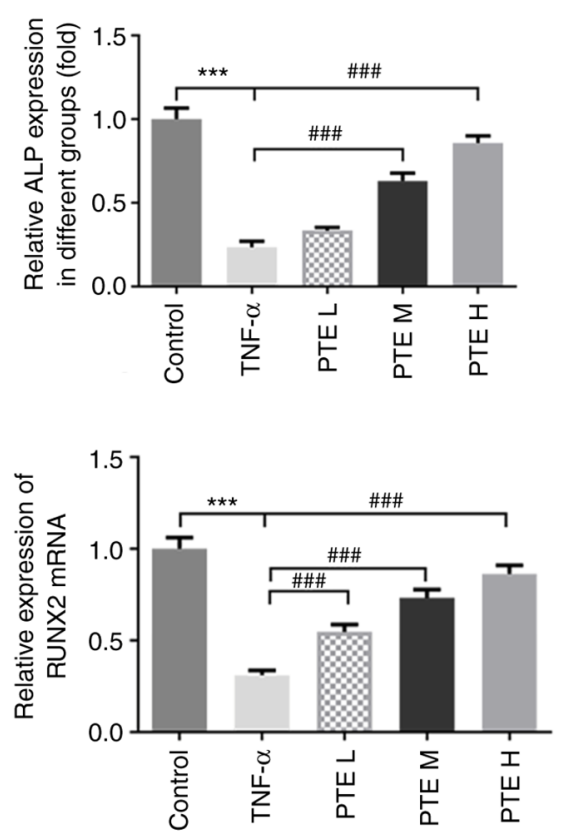

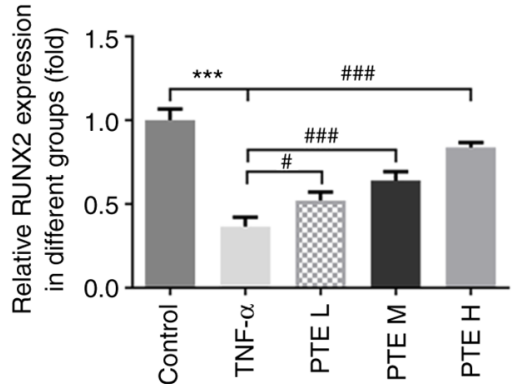

D

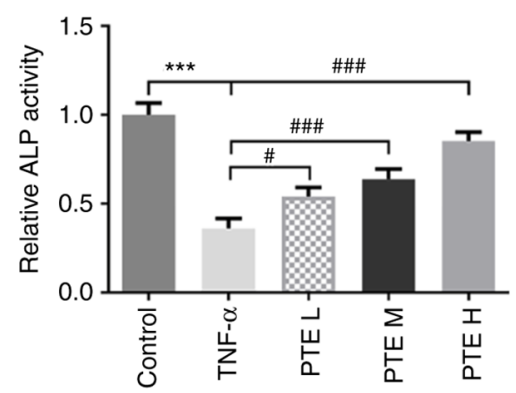

Figure 3. PTE promotes TNF- $\alpha$-induced hPDLSCs differentiation. (A) Role of PTE in human periodontal ligament stem cell mineralization was evaluated using alizarin red staining. (B) The protein expression levels of ALP and RUNX2 were determined using western blotting. (C) The mRNA expression levels of ALP and RUNX2 were determined using RT-qPCR. (D) The activity of ALP was assessed using a detection kit. ${ }^{* * *} \mathrm{P}<0.001 ;{ }^{*} \mathrm{P}<0.05$, ${ }^{\# \#} \mathrm{P}<0.01$ and ${ }^{\# \# \#} \mathrm{P}<0.001$. PTE, pterostilbene; ALP, alkaline phosphatase; RUNX2, runt-related transcription factor 2; L, low dose; M, medium dose; H, high dose.

8 were $-6.0,-7.3,-6.4$ and $-6.4 \mathrm{kcal} / \mathrm{mol}$, respectively, which indicated that the complex formation is thermodynamically spontaneous and implied the high stability of PTE-HDAC2, 4, 6 and 8 complexes (Fig. 4). Following computer prediction, the expression levels of these four proteins were determined using western blotting. TNF- $\alpha$ significantly increased the expression levels of all four of the HDAC isoforms tested, whilst PTE treatment markedly decreased HDAC4, 6 and 8 expression in a concentration-dependent manner compared with the TNF- $\alpha$ group. However, no significant difference could be observed for HDAC2 between the TNF- $\alpha$ and PTE groups (Fig. 5).

\section{Discussion}

hPDLSCs derived from human periodontal tissues are a type of mesenchymal stem cell that is readily obtainable in clinical practice (28). They can be obtained following wisdom teeth extraction or premolars that are extracted by orthodontists using minimally invasive methods (29). This availability render hPDLSCs attractive sources of autologous stem cells. A previous study reported that the addition of hPDLSCs to biological materials is beneficial to promote bone healing in rat skull defect models (30). In addition, a number of in vitro and in vitro studies have demonstrated that hPDLSCs exhibit potent self-renewal and multi-lineage differentiation capacities $(31,32)$. Their ability to differentiate into osteoblasts makes them considerably superior options to stem cells derived from other oral tissues, including the dental pulp, gums and dental follicles (33). These characteristics also mean that hPDLSCs have been extensively applied for the regeneration treatment of periodontal disease-induced periodontal cord and bone loss (12). Therefore, the present study selected hPDLSCs to assess the possible role of PTE in periodontitis, which 
A
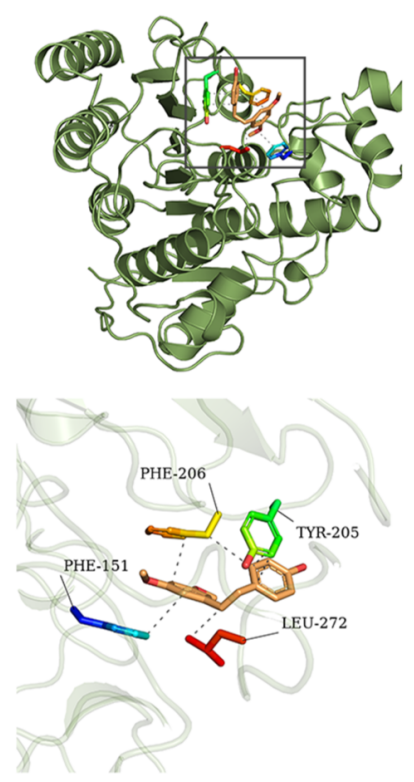

HDAC2-PTE
B
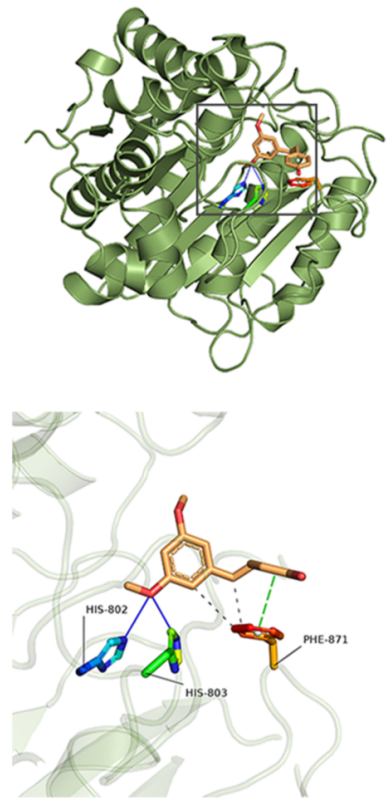

HDAC4-PTE
C
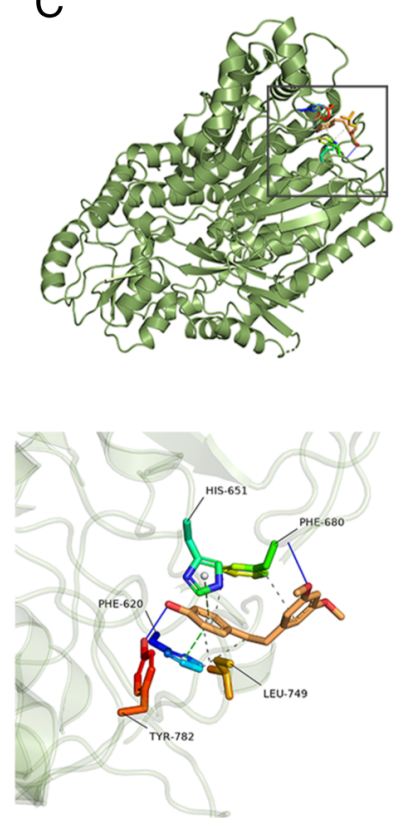

HDAC6-PTE
D
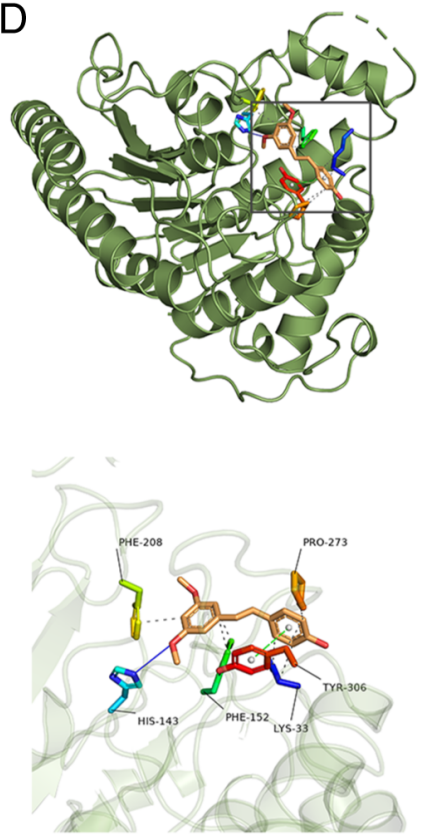

HDAC8-PTE

Figure 4. Molecular docking results of PTE with HDACs. The full field of view and enlarged partial fields of view of PTE with (A) HDAC2,(B) HDAC4,(C) HDAC6 and (D) HDAC8. The whole protein was presented in green, whilst the main skeleton of PTE was presented in brown. The blue solid line represented hydrogen bonds, the green dashed line represented $\pi$-stacking and the gray dashed line represent hydrophobic interactions. PTE, pterostilbene; HDAC, histone deacetylase.
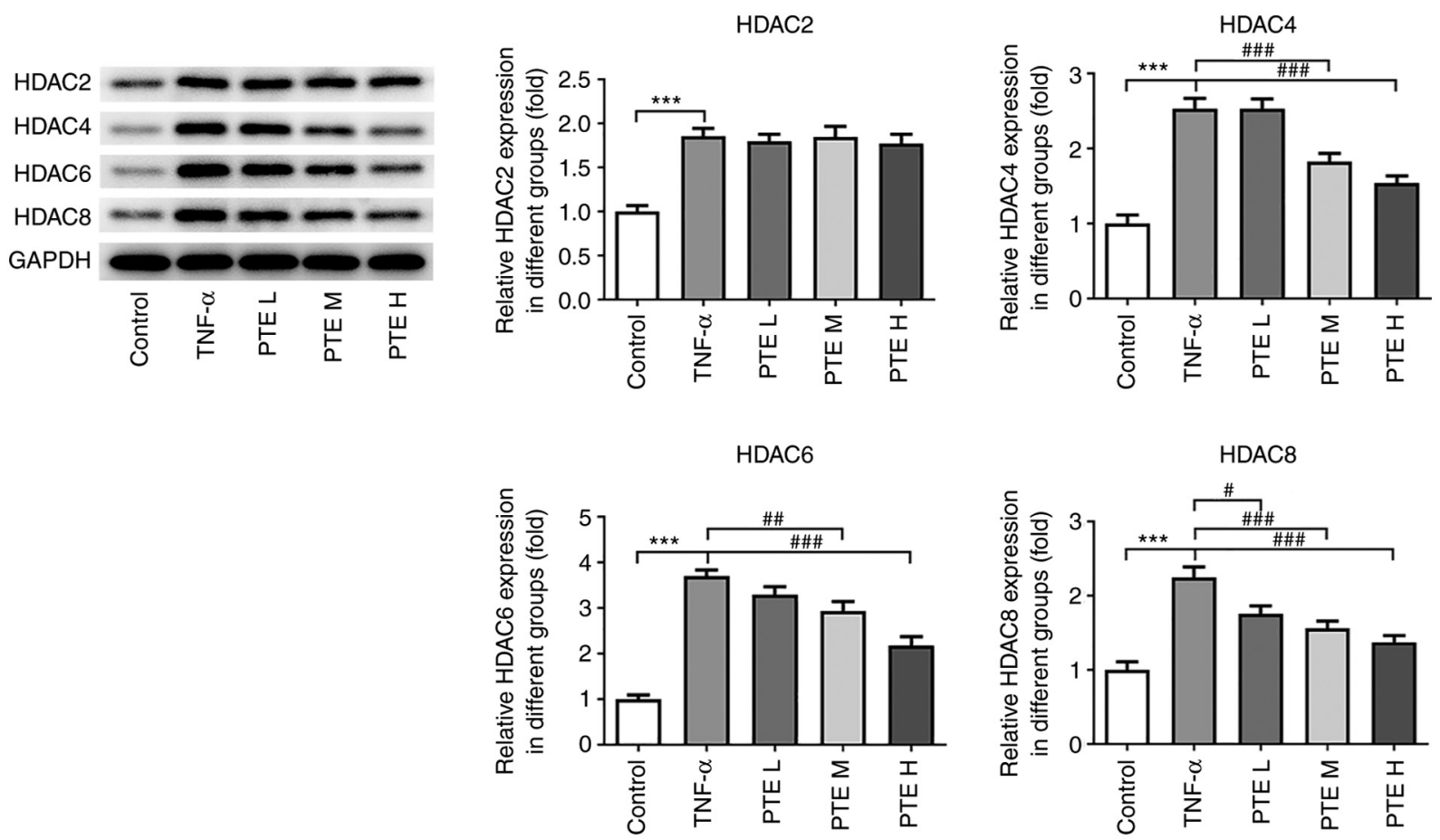

Figure 5. Expression levels of HDAC2, 4, 6 and 8 were determined using western blotting. ${ }^{* * *} \mathrm{P}<0.001 ;{ }^{\#} \mathrm{P}<0.05$, ${ }^{\# \#} \mathrm{P}<0.01$ and ${ }^{\# \# \#} \mathrm{P}<0.001$. HDAC, histone deacetylase; L, low dose; $\mathrm{M}$, medium dose; $\mathrm{H}$, high dose.

revealed that PTE increased the viability of hPDLSCs in a concentration-dependent manner.

During the progression of periodontitis, the increased expression of TNF- $\alpha$ further promotes the secretion of other inflammatory mediators, adhesion molecules and cytokines that are closely associated with bone resorption (22). This aggravates the clinical symptoms of periodontitis (22). TNF- $\alpha$ was used in the present study to treat hPDLSCs, after which the effects of PTE on the cell viability, inflammatory factor release, apoptosis and differentiation of hPDLSCs were assessed. PTE 
was revealed to upregulate the expression levels of proliferation markers Ki67 and PCNA, and reduce IL-1 $\beta$ and IL-6 levels, whilst increasing IL-10 levels. It has been previously demonstrated that IL-10 serves an anti-inflammatory role by strongly inhibiting the synthesis of IL-1, IL-6, IL- 8 and TNF- $\alpha$ on a transcriptional level, regardless of whether it is endogenous or exogenous (34-36). The results of the present study revealed that PTE increased the viability of TNF- $\alpha$-induced hPDLSCs and reduced the release of inflammatory cytokines. In addition, results from TUNEL staining and western blotting demonstrated that PTE significantly inhibited the apoptosis of TNF- $\alpha$-induced hPDLSCs. A previous study also revealed that PTE inhibited inflammation and apoptosis in mouse models of pulmonary fibrosis (37). ALP has been considered to be an early osteogenic marker, whilst RUNX2 regulates the differentiation of pluripotent stem cells into osteoblasts (38). In the present study, PTE significantly upregulated the expression of ALP and RUNX2 on day 7 of osteogenic induction in TNF- $\alpha$-induced hPDLSCs, suggesting that PTE promoted hPDLSC differentiation.

Using the Targetnet database, PTE was predicted to target histone family protein HDACs. Previous studies have revealed that HDAC inhibitors can promote the proliferation of human dental pulp stem cells and the differentiation of odontoblasts $(39,40)$. Furthermore, HDAC2 inhibition has been demonstrated to reduce cytokine production and osteoclast bone resorption (41). A previous study also reported that microRNA-22 targets HDAC6 to promote hPDLSC differentiation (42). In the present study, according to computer simulation software, it was determined that PTE could bind to HDACs through intermolecular forces. Additionally, the docking value between PTE and HDAC4 was the most optimal among all HDACs. Western blotting revealed that PTE treatment significantly downregulated the protein expression levels of HDAC4, 6 and 8, with HDAC4 and 6 exhibiting relatively higher concentration-dependence. These results suggest that PTE may participate in the regulation of periodontitis by downregulating HDAC expression. The downregulation of HDACs reduces the removal of acetylated groups on the lysine residues of histones, destabilizing the binding between histones and DNA (43). This detachment promotes the combination of the DNA promoter regions with transcription factors (44), which increases the transcription of genes related to proliferation and differentiation $(45,46)$. This may be the mechanism through which PTE can regulate proliferation and differentiation via HDACs. However, results of the present study are limited to the preliminary exploration of this mechanism. Additional experiments are required to verify the association between PTE and HDACs in periodontitis. Furthermore, in addition to osteogenic differentiation, cartilage and adipogenic multidirectional differentiation are also important indices of the differentiation potential of stem cells. Future studies on the effects of PTE on these two aspects also warrant further investigation.

In conclusion, the present study revealed that PTE promoted TNF- $\alpha$-induced hPDLSC viability and differentiation, whilst alleviating inflammation and apoptosis. PTE also inhibited the expression of HDACs, which may be its mechanism of action in periodontitis. It is hoped that findings of the present study may promote the development of novel treatment strategies for periodontitis.

\section{Acknowledgements}

Not applicable.

\section{Funding}

No funding was received.

\section{Availability of data and materials}

The datasets used and/or analyzed during the current study are available from the corresponding author on reasonable request.

\section{Authors' contributions}

MY, GW and YL conceived this study. MY, GW and JN performed the experiments. MP analyzed the data and YL performed bioinformatics analysis. YL revised the manuscript for important intellectual content. All authors have read and approved the final manuscript. MY and GW confirm the authenticity of the raw data.

\section{Ethics approval and consent to participate}

The Ethics Committee of Shanghai Huangpu District 2nd Dental Disease Prevention and Treatment Institute (Shanghai, China) waived the requirement for ethics approval for using the purchased human periodontal ligament stem cells.

\section{Patient consent for publication}

Not applicable.

\section{Competing interests}

The authors declare that they have no competing interests.

\section{References}

1. Hoare A, Soto C, Rojas-Celis V and Bravo D: Chronic inflammation as a link between periodontitis and carcinogenesis. Mediators Inflamm 2019: 1029857, 2019.

2. Gasner NS and Schure RS: Periodontal disease. In: StatPearls. StatPearls Publishing, Treasure Island, FL, 2021.

3. Liu J, Ruan J, Weir MD, Ren K, Schneider A, Wang P, Oates TW, Chang $\mathrm{X}$ and $\mathrm{Xu}$ HHK: Periodontal bone-ligament-cementum regeneration via scaffolds and stem cells. Cells 8: 537, 2019.

4. Graziani F, Karapetsa D, Alonso B and Herrera D: Nonsurgical and surgical treatment of periodontitis: How many options for one disease? Periodontol 2000 75: 152-188, 2017.

5. Deas DE, Moritz AJ, Sagun RS Jr, Gruwell SF and Powell CA: Scaling and root planing vs. conservative surgery in the treatment of chronic periodontitis. Periodontol 2000 71: 128-139, 2016.

6. Reynolds MA, Kao RT, Camargo PM, Caton JG, Clem DS, Fiorellini JP, Geisinger ML, Mills MP, Nares S and Nevins ML: Periodontal regeneration-intrabony defects: A consensus report from the AAP regeneration workshop. J Periodontol 86 (Suppl 2): S105-S107, 2015.

7. Rojas MA, Marini L, Pilloni A and Sahrmann P: Early wound healing outcomes after regenerative periodontal surgery with enamel matrix derivatives or guided tissue regeneration: A systematic review. BMC Oral Health 19: 76, 2019. 
8. Gao F, Chiu SM, Motan DA, Zhang Z, Chen L, Ji HL, Tse HF, Fu QL and Lian Q: Mesenchymal stem cells and immunomodulation: Current status and future prospects. Cell Death Dis 7: e2062, 2016

9. Moradi SL, Golchin A, Hajishafieeha Z, Khani MM and Ardeshirylajimi A: Bone tissue engineering: Adult stem cells in combination with electrospun nanofibrous scaffolds. J Cell Physiol 233: 6509-6522, 2018.

10. Ouchi T and Nakagawa T: Mesenchymal stem cell-based tissue regeneration therapies for periodontitis. Regen Ther 14: 72-78, 2020.

11. Yoshida T, Washio K, Iwata T, Okano T and Ishikawa I: Current status and future development of cell transplantation therapy for periodontal tissue regeneration. Int J Dent 2012: 307024, 2012.

12. Huang GT, Gronthos S and Shi S: Mesenchymal stem cells derived from dental tissues vs. those from other sources: Their biology and role in regenerative medicine. J Dent Res 88: 792-806, 2009.

13. Pecyna P, Wargula J, Murias $M$ and Kucinska M: More than resveratrol: New insights into stilbene-based compounds. Biomolecules 10: 1111, 2020

14. Cassiano C, Eletto D, Tosco A, Riccio R, Monti MC and Casapullo A: Determining the effect of pterostilbene on insulin secretion using chemoproteomics. Molecules 25: 2885, 2020.

15. Kim H, Seo KH and Yokoyama W: Chemistry of pterostilbene and its metabolic effects. J Agric Food Chem 68: 12836-12841, 2020.

16. Lange KW and Li S: Resveratrol, pterostilbene, and dementia. Biofactors 44: 83-90, 2018

17. Ma Z, Zhang X, Xu L, Liu D, Di S, Li W, Zhang J, Zhang H, Li X, Han J and Yan X: Pterostilbene: Mechanisms of its action as oncostatic agent in cell models and in vivo studies. Pharmacol Res 145: 104265, 2019.

18. Guo Y, Zhang L, Li F, Hu CP and Zhang Z: Restoration of sirt1 function by pterostilbene attenuates hypoxia-reoxygenation injury in cardiomyocytes. Eur J Pharmacol 776: 26-33, 2016.

19. Wu M, Lu S, Zhong J, Huang K and Zhang S: Protective effects of pterostilbene against myocardial ischemia/reperfusion injury in rats. Inflammation 40: 578-588, 2017.

20. Zhang Y and Zhang Y: Pterostilbene, a novel natural plant conduct, inhibits high fat-induced atherosclerosis inflammation via NF- $\kappa \mathrm{B}$ signaling pathway in Toll-like receptor 5 (TLR5) deficient mice. Biomed Pharmacother 81: 345-355, 2016.

21. Lim YRI, Preshaw PM, Lim LP, Ong MMA, Lin HS and Tan KS Pterostilbene complexed with cyclodextrin exerts antimicrobial and anti-inflammatory effects. Sci Rep 10: 9072, 2020.

22. Kitaura H, Kimura K, Ishida M, Kohara H, Yoshimatsu M and Takano-Yamamoto $\mathrm{T}$ : Immunological reaction in TNF- $\alpha$-mediated osteoclast formation and bone resorption in vitro and in vivo. Clin Dev Immunol 2013: 181849, 2013

23. Liu H, Wu X, Luo J, Wang X, Guo H, Feng D, Zhao L, Bai H, Song M, Liu X, et al: Pterostilbene attenuates astrocytic inflammation and neuronal oxidative injury after ischemia-reperfusion by inhibiting NF- $\kappa$ B phosphorylation. Front Immunol 10: 2408 , 2019.

24. Meng T, Zhou Y, Li J, Hu M, Li X, Wang P, Jia Z, Li L and Liu D Azithromycin promotes the osteogenic differentiation of human periodontal ligament stem cells after stimulation with TNF- $\alpha$. Stem Cells Int 2018: 7961962, 2018

25. Yao ZJ, Dong J, Che YJ, Zhu MF, Wen M, Wang NN, Wang S, Lu AP and Cao DS: TargetNet: A web service for predicting potential drug-target interaction profiling via multi-target SAR models. J Comput Aided Mol Des 30: 413-424, 2016.

26. Li J, Fu A and Zhang L: An overview of scoring functions used for protein-ligand interactions in molecular docking. Interdiscip Sci 11: 320-328, 2019.

27. Livak KJ and Schmittgen TD: Analysis of relative gene expression data using real-time quantitative PCR and the 2(-Delta Delta C(T)) method. Methods 25: 402-408, 2001

28. Xia Y, Tang HN, Wu RX, Yu Y, Gao LN and Chen FM: Cell responses to conditioned media produced by patient-matched stem cells derived from healthy and inflamed periodontal ligament tissues. J Periodontol 87: e53-e63, 2016.
29. Chen FM, Sun HH, Lu H and Yu Q: Stem cell-delivery therapeutics for periodontal tissue regeneration. Biomaterials 33: 6320-6344, 2012

30. Pizzicannella J, Gugliandolo A, Orsini T, Fontana A, Ventrella A, Mazzon E, Bramanti P, Diomede F and Trubiani O: Engineered extracellular vesicles from human periodontal-ligament stem cells increase VEGF/VEGFR2 expression during bone regeneration. Front Physiol 10: 512, 2019.

31. Bright R, Hynes K, Gronthos S and Bartold PM: Periodontal ligament-derived cells for periodontal regeneration in animal models: A systematic review. J Periodontal Res 50: 160-172, 2015.

32. Zhang Y, Xing Y, Jia L, Ji Y, Zhao B, Wen Y and Xu X: An in vitro comparative study of multisource derived human mesenchymal stem cells for bone tissue engineering. Stem Cells Dev 27: 1634-1645, 2018

33. Trubiani O, Pizzicannella J, Caputi S, Marchisio M, Mazzon E, Paganelli R, Paganelli A and Diomede F: Periodontal ligament stem cells: Current knowledge and future perspectives. Stem Cells Dev 28: 995-1003, 2019.

34. Fang D and Zhu J: Molecular switches for regulating the differentiation of inflammatory and IL-10-producing anti-inflammatory T-helper cells. Cell Mol Life Sci 77: 289-303, 2020.

35. Munshi S, Parrilli V and Rosenkranz JA: Peripheral anti-inflammatory cytokine Interleukin-10 treatment mitigates interleukin-1 $\beta$-induced anxiety and sickness behaviors in adult male rats. Behav Brain Res 372: 112024, 2019.

36. Ng A, Tam WW, Zhang MW, Ho CS, Husain SF, McIntyre RS and Ho RC: IL-1 $\beta$, IL-6, TNF- $\alpha$ and CRP in elderly patients with depression or Alzheimer's disease: Systematic review and meta-analysis. Sci Rep 8: 12050, 2018

37. Yang H, Hua C, Yang X, Fan X, Song H, Peng L and Ci X: Pterostilbene prevents LPS-induced early pulmonary fibrosis by suppressing oxidative stress, inflammation and apoptosis in vivo. Food Funct 11: 4471-4484, 2020

38. Newton AH and Pask AJ: CHD9 upregulates RUNX2 and has a potential role in skeletal evolution. BMC Mol Cell Biol 21: 27, 2020.

39. Jin H, Park JY, Choi $\mathrm{H}$ and Choung PH: HDAC inhibitor trichostatin A promotes proliferation and odontoblast differentiation of human dental pulp stem cells. Tissue Eng Part A 19: 613-624, 2013.

40. Liu Z, Chen T, Han Q, Chen M, You J, Fang F, Peng L and Wu B: HDAC inhibitor LMK-235 promotes the odontoblast differentiation of dental pulp cells. Mol Med Rep 17: 1445-1452, 2018.

41. Algate K, Haynes D, Fitzsimmons T, Romeo O, Wagner F, Holson E, Reid R, Fairlie D, Bartold P and Cantley M: Histone deacetylases 1 and 2 inhibition suppresses cytokine production and osteoclast bone resorption in vitro. J Cell Biochem 121: 244-258, 2020

42. Yan GQ, Wang X, Yang F, Yang ML, Zhang GR, Wang GK and Zhou Q: MicroRNA-22 promoted osteogenic differentiation of human periodontal ligament stem cells by targeting HDAC6. J Cell Biochem 118: 1653-1658, 2017.

43. Leonova K, Safina A, Nesher E, Sandlesh P, Pratt R, Burkhart C, Lipchick B, Gitlin I, Frangou C, Koman I, et al: TRAIN (Transcription of Repeats Activates INterferon) in response to chromatin destabilization induced by small molecules in mammalian cells. Elife 7: e30842, 2018.

44. Elmer JJ, Christensen MD, Barua S, Lehrman J, Haynes KA and Rege K: The histone deacetylase inhibitor entinostat enhances polymer-mediated transgene expression in cancer cell lines. Biotechnol Bioeng 113: 1345-1356, 2016.

45. Liao XH,Lu DL, Wang N,Liu LY, Wang Y,Li YQ, Yan TB, Sun XG, $\mathrm{Hu} \mathrm{P}$ and Zhang TC: Estrogen receptor $\alpha$ mediates proliferation of breast cancer MCF-7 cells via a p21/PCNA/E2F1-dependent pathway. FEBS J 281: 927-942, 2014.

46. Zhang Q, Zuo H, Yu S, Lin Y, Chen S, Liu H and Chen Z: RUNX2 co-operates with EGR1 to regulate osteogenic differentiation through Htral enhancers. J Cell Physiol 235: 8601-8612, 2020.

This work is licensed under a Creative Commons Attribution-NonCommercial-NoDerivatives 4.0 International (CC BY-NC-ND 4.0) License. 